\title{
Modell des Primärprozesses beim Sehvorgang an der Reizschwelle
}

\author{
Von F. J. Esper \\ Aus dem Institut für Strahlen- und Kernphysik, Bonn \\ (Z. Naturforschg. 9 b, 13-16 [1954]; eingegangen am 2. September 1953)
}

\begin{abstract}
Es wird eine Modellvorstellung entwickelt, die versucht, die physikalisch-chemische PrimärReaktion beim Sehvorgang zu erklären. Dazu werden die extrafovealen Sehzellen in Analogie zu einer Sperrschichtphotozelle gesetzt. Zur Erklärung der gleichen Reaktion bei den fovealen Sehzellen reicht dieses einfache Modell nicht aus.
\end{abstract}

A uf Grund von Messungen an der Reizschwelle des menschlichen Auges soll im Folgenden ein Modell des Primärprozesses beim Sehvorgang entwickelt werden. Bei dem Versuch, wenigstens die Primär-Reaktion durch eine physikalische Gesetzmäßigkeit zu erklären, und bei der Beurteilung dieses Vorhabens muß man sich stets vergegenwärtigen, daß man die physikalisch-chemische Primär-Reaktion nicht isoliert am lebenden Auge untersuchen kann, sondern daß der Sehvorgang nur in seiner Gesamtheit dem Experiment zugänglich ist. Dabei spielen aber Vorgänge in den höheren Zentren eine wichtige Rolle, deren Ablauf nicht durch eine Formel beschrieben werden kann.

\section{Ergebnis se}

Es wurde die Reizschwelle des menschlichen Auges in Abhängigkeit von der Wellenlänge des einfallenden Lichtes untersucht, und zwar einmal für einen extrafovealen Bereich, der $8^{\circ}$ oberhalb der Fovea centralis lag, und zum anderen für einen in der Fovea centralis gelegenen Bereich. (Näheres siehe ${ }^{7}$.)

Die Ergebnisse sind in Abb. 1 und Abb. 2 graphisch wiedergegeben. Abb. 1 zeigt die Ergebnisse, die für die Empfindlichkeit der extrafovealen Sehzellen ermittelt wurden. Auf der Abszisse sind die Lichtfrequenzen aufgetragen. Die Ordinate gibt die Zahl der notwendig absorbierten Quanten an. Daneben ist die bei den Quantenabsorptionen aufgenommene Energie (in erg) in Abhängigkeit von der Frequenz des einfallenden Lichtes dargestellt. Abb. 2 gibt das Entsprechende für die Fovea centralis wieder.

Es sei hier kurz auf die Vorstellung hingewiesen, die man sich vom Zustandekommen des Sehvorganges macht:

Durch die Absorption eines Lichtquants in den Sehzellen der Retina wird im Auge eine physikalisch- chemische Primär-Reaktion ausgelöst, welche die Ursache für einen Reiz darstellt. Über verschiedene Schaltelemente erfolgt die Reizleitung zum Gehirn und dort die Umwandlung des Reizes in die Sinneswahrnehmung, die wir als Sehen bezeichnen.

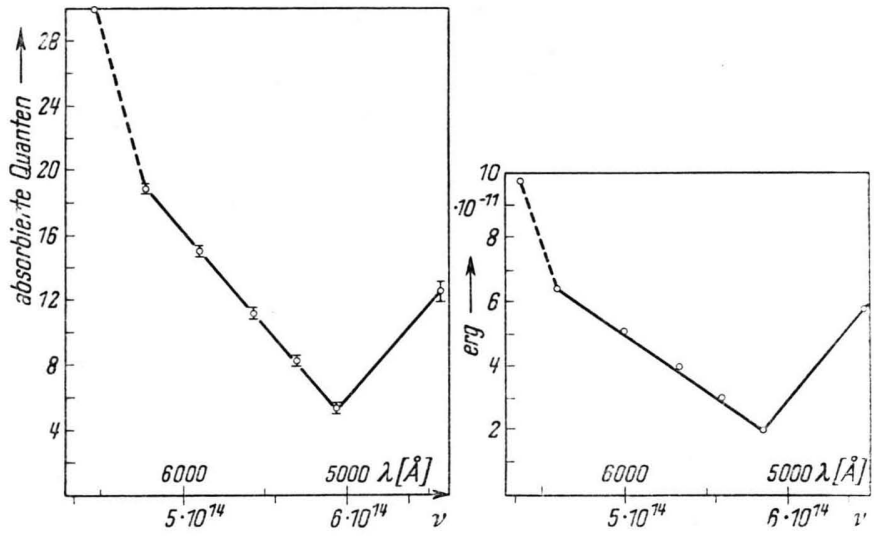

Abb. 1. Anzahl der Lichtquanten und die ihnen entsprechende Energie (in erg), die notwendig absorbiert werden müssen, um eine Sehwahrnehmung hervorzurufen; beide Werte sind in Abhängigkeit von der Frequenz des einfallenden Lichtes aufgetragen. Die Darstellung gilt für einen extrafovealen Bereich, der $8^{\circ}$ oberhalb der Fovea centralis liegt.

Bisher hat man nun angenommen, daß das Eintreten einer Sehwahrnehmung nur vom Eintreffen einer bestimmten Anzahl von Quantenabsorptionen abhängig sei, wobei die Anzahl der notwendigerweise zu absorbierenden Quanten unabhängig von der Wellenlänge des einfallenden Lichtes sei ${ }^{1,} \stackrel{2}{ }$.

Dieser Annahme widerspricht aber obiges Ergebnis für die extrafovealen Sehzellen ganz entschieden. Auch für die in der Fovea centralis gelegenen Seh-

1 E. B a u m g a r d t, Naturwissenschaften $\quad 39, \quad 388$ [1952].

2 M. H. Pirenne, Progr. Biophysics 2 [1951]. 
elemente ist diese Forderung nach den Ergebnissen der Abb. 2 nicht ohne Zusatzannahmen haltbar.

Man könnte nun fordern, daß eine von der Wellenlänge unabhängige, immer gleich große Energiemenge, die in den Sehzellen absorbiert wird, die Sehwahrnehmung auslösen könnte. Die Minima der beiden Energiekurven (s. Abb. 1 und Abb. 2) lassen aber auch dieses Postulat als unrichtig erscheinen.

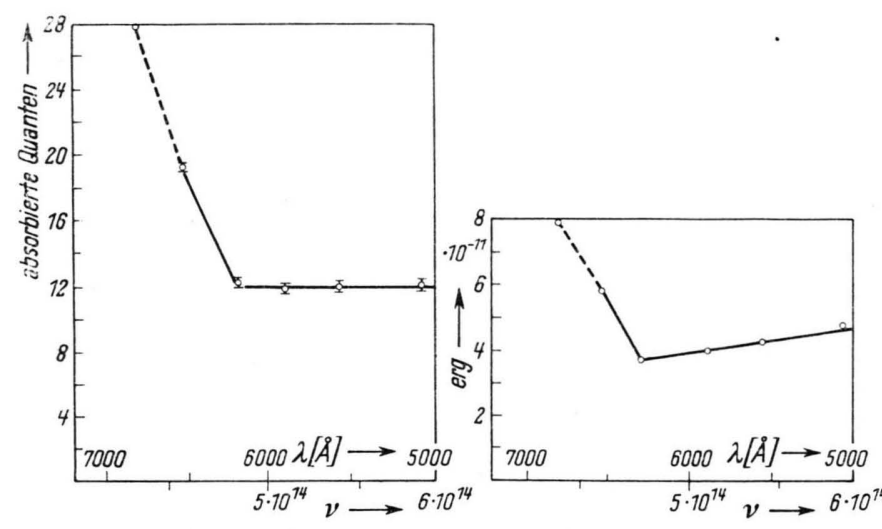

Abb. 2. Die der Abb. 1 entsprechenden Ergebnisse für die Fovea centralis.

Die Modellvorstellung

Die besondere Schwierigkeit der theoretischen Deutung liegt neben den schon angedeuteten Schwierigkeiten darin, das Minimum der notwendig absorbierten Quanten bei $\lambda=5050 \AA$ für die Stäbchen zu erklären.

Ich möchte nun die Sehzellen der Retina, zunächst nur die extrafovealen zu einer Sperrschichtphotozelle in Analogie setzen. Voraussetzung dazu ist allerdings, daß der Sehpurpur in kristalliner Form in den Schzellen vorliegt. Diese Annahme ist nach A utrum durchaus berechtigt ${ }^{3}$. Den kristallinen Sehpurpur kann man dann als Halbleiter betrachten.

Lehovec hat eine Theorie über den SperrschichtPhotoeffekt gegeben, die sich auf Arbeiten von Schottky und Mott stützt. Aus dieser Theorie möchte ich nur die hier interessierenden Punkte anführen ${ }^{4-6}$.

Bei der Sperrschicht-Photozelle - hier wird eine sog. Vorderwandzelle betrachtet - ist auf eine Metallelektrode $\mathrm{M}_{1}$ eine Halbleiterschicht aufgebracht. Darauf befindet sich eine zweite Metallelektrode $\mathrm{M}_{2}$, die lichtdurchlässig ist. Die Sperrschicht bildet sich

3 H. J. A utrum, Naturwissenschaften 39, 290 [1952].

4 K. Le h ove c, Z. Naturforschg. 1, 258 [1946].

5 K. L e hovec, Z. Naturforschg. 2, 398 [1947]. in der unmittelbar an die Elektrode $\mathrm{M}_{2}$ grenzenden Schicht im Halbleiter aus. Die Bildung einer Sperrschicht an der Elektrode $\mathbf{M}_{1}$ kann man verhindern. Bei einer Belichtung des Halbleiters durch $\mathrm{M}_{2}$ hindurch liegt zwischen $\mathrm{M}_{1}$ und $\mathrm{M}_{2}$ eine Spannung, die sog. Photospannung.

Der Halbleiter ist im ganzen elektrisch neutral. In der Sperrschicht aber nimmt die Dichte der DefektElektronen bzw. der Elektronen ab, je nachdem ob es sich um einen Defekt-Halbleiter oder ÜberschußHalbleiter handelt. Dem Diffusions-Potential, bedingt durch das Konzentrations-Gefälle der Defekt-Elektronen in der Sperrschicht, wird durch ein elektrisches Potential das Gleichgewicht gehalten. Wird dieses Gleichgewicht durch Belichtung der Photozelle gestört, so tritt ein resultierender Spannungsabfall nach außen in Erscheinung. Seine Größe ist:

$$
E=V_{R}-\mathfrak{B} \cdot \ln \left(n_{\mathrm{d}} / n_{\mathrm{R}}\right) .
$$

$V_{R}=$ elektrischer Spannungsabfall an der Sperrschicht, $\mathfrak{B}=k T / e=$ die der Temperatur äquivalente Spannung, $n_{d}, n_{R}=$ Dichte der Defektelektronen am halbleiterseitigen bzw. metallseitigen Rand der Sperrschicht. (Im folgenden soll ein Defekt-Halbleiter betrachtet werden.) $e=$ Elementarladung.

Das an der Sperrschicht liegende Potential, dessen Höchstwert am metallseitigen Rand erreicht wird, läßt sich berechnen.

Im stationären Zustand fließen alle bei Belichtung ausgelösten Defekt-Elektronen ab, da bei der geringen Defekt-Elektronendichte kaum Rekombinationen auftreten. Für den Bereich der Sperrschicht ergibt sich deshalb:

$$
Z \cdot \frac{1}{x_{0}} \exp \left(-\frac{x}{x_{0}}\right)-\begin{array}{ll}
1 & \mathrm{~d} i \\
e & \mathrm{~d} x
\end{array}=0 .
$$

$Z=$ Gesamtzahl der pro $\mathrm{cm}^{2}$ und sec einfallenden Lichtquanten,

$x_{0}=$ Absorptionslänge des Halbleiters,

$x=$ Raumkoordinate senkrecht zur Oberfläche der Photozelle, zunehmend gegen den Halbleiter $(x=0=$ metallseitiger Rand der Sperrschicht, $x=d=$ halbleiterseitiger Rand).

Als Beziehung zwischen Spannung an der Sperrschicht, dem Defekt-Elektronenstrom und der Zahl der einfallenden Lichtquanten gibt Leho v e c schließlich an:

${ }^{6}$ K. Lehovec, Physic. Rev. 74, 1; 463 [1948].

; W. Riezler, F. J. Esper u. H. Meurers, [im Druck]. 


$$
\begin{aligned}
Z e\left[\left(1+\frac{\mathfrak{B}}{巨_{\mathrm{r}} x_{0}}\right)^{-1}-\exp \left(-\frac{d}{x_{0}}\right)\right]-I \\
=n_{R} e b \xi_{\mathrm{r}}\left[\exp \left(-\begin{array}{c}
E \\
\mathfrak{D}
\end{array}\right)-1\right] .
\end{aligned}
$$

Darin bedeuten: $\mathfrak{夭}_{\mathrm{r}}=$ Feldstärke an der Elektrode, $b=$ Beweglichkeit der Defekt-Elektronen, $I=\mathrm{Ge}-$ samtstrom durch die Photozelle, $d=$ Dicke der Sperrschicht.

Lehovec bestimmt nun aus Gl. (3) die spektrale Verteilung der Quantenausbeute des Kurzschlußstromes. Es ist dann $E=0$. Unter Quantenausbeute

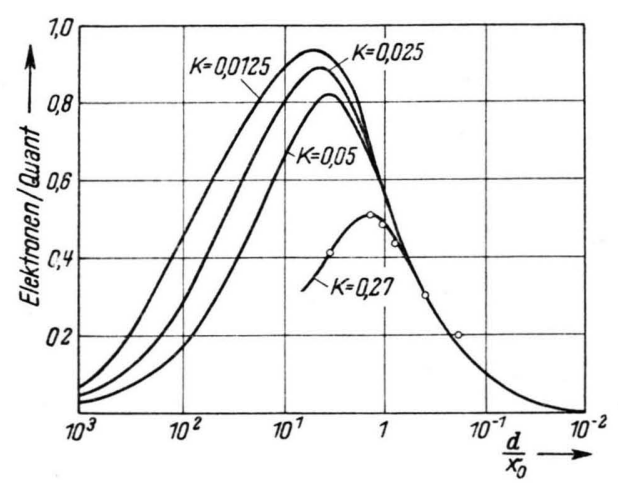

Abb. 3. Spektrale Verteilung der Quantenausbeute. Die Absorptionslänge $x_{0}$ ist eine Funktion der Wellenlänge des einfallenden Lichtes. Die Kurven mit den Parametern $K=0,0125, K=0,025$ und $K=0,05$ gelten für eine Selensperrschicht-Photozelle, während die ihnen entsprechende Kurve mit dem Parameter $K=0,27$ für das Auge berechnet ist.

versteht man das Verhältnis Elektron/Quant. Es ergibt sich mit $E=0$ :

$$
Z e\left[\left(1+\frac{\mathfrak{B}}{\xi_{\mathrm{r}} x_{0}}\right)^{-1}\right]-\exp \left(-\frac{d}{x_{0}}\right)=I .
$$

Daraus folgt für die Quantenausbeute der Ausdruck:

$$
\delta=\frac{I}{Z e}=\left[\left(1+\frac{\mathfrak{B}}{\Xi_{\mathrm{r}} x_{0}}\right)^{-1}\right]-\exp \left(-\begin{array}{c}
d \\
x_{0}
\end{array}\right) .
$$

In Abb. 3 sind einige Kurven für die spektrale Verteilung der Quantenausbeute für verschiedene Werte des Parameters $K=\mathfrak{B} / \mathfrak{E}_{\mathrm{r}} d$ in Abhängigkeit von $d / x_{0}$ eingezeichnet. Die Absorptionslänge $x_{0}$ ist eine Funktion der Wellenlänge des einfallenden Lichtes

$$
\left[x_{0}=x_{0}(\lambda)\right] \text {. }
$$

Lehovec kommentiert diese Kurven folgendermaßen: „Das Maximum der Ausbeute liegt bei Absorptionslängen, die einige Zehntel der Sperrschicht-
Dicke betragen: Fast alle durch das Licht ausgelösten Defekt-Elektronen werden durch das elektrische Feld in den Halbleiter geführt. Der Abfall der Ausbeute gegen große Absorptionslängen $\left(x_{0}>d\right.$, lange Wellen) beruht auf der geringen Absorption des Lichtes innerhalb der Sperrschicht. Der Abfall gegen kleine Absorptionslängen $\left(x_{0}<d\right.$, kurze Wellen) kommt dadurch zustande, daß sich ein zu starkes Konzentrations-Gefälle in der Sperrschicht ausbildet, wodurch ein Teil der Defekt-Elektronen zur Elektrode getrieben wird."

Die Kurven der Abb. 3 (ausgenommen die Kurve mit dem Parameter $K=0,27$ ) gelten für eine Selenphoto-Sperrschichtzelle. Ich habe nun unter ganz bestimmten Annahmen eine entsprechende Kurve für das Auge berechnet. Diese spektrale Verteilungskurve mußte das Maximum der Quantenausbeute bei $\lambda=5050 \AA$ haben. Diese Bedingung legte die zu treffenden Annahmen schon weitgehend fest.

Folgendes sind die Annahmen, die der Rechnung zugrunde liegen: $\mathfrak{B}=k T / e$ ist durch die Körpertemperatur bestimmt. Die Sperrschichtdicke $d$ habe ich mit $10^{-5} \mathrm{~cm}$ angenommen. Dies ist die gleiche Größe wie bei der Selen-Photozelle. Die Feldstärke $\mathfrak{F}_{r}$ am metallseitigen Rand der Sperrschicht soll $10^{4} \mathrm{Volt} / \mathrm{cm}$ betragen gegenüber $10^{5}$ Volt $/ \mathrm{cm}$ beim Selen. Die Absorptionslänge $x_{0}$ soll von $10^{-6} \mathrm{~cm}$ bei $\lambda=4000 \AA$ linear auf $x_{0}=10^{-4} \mathrm{~cm}$ bei $\lambda=7000 \AA$ zunehmen. Das ist ungefähr die gleiche Größenordnung wie bei der Selen-Photozelle.

Die Richtigkeit der Annahme über die Feldstärke $\mathfrak{E}_{\mathrm{r}}$ und die Sperrschicht-Dicke $d$ bestätigt A utrum ${ }^{3}$, w€nn er schreibt: „In lebenden Zellen können elektrische Felder beträchtlicher Stärke auftreten: Über Doppelschichten, die aus wenigen Molekel-Lagen bestehen, wurden Potentiale von $100 \mathrm{mV}$ und mehr gemessen." Bei einem Durchmesser der Farbstoffmoleküle von der Größenordnung von $10^{-6} \mathrm{~cm}$ entsprechen der Sperrschichtdicke $d$ von $10^{-5} \mathrm{~cm} 10$ nebeneinanderliegende Moleküle. Der Spannungsabfall über der Sperrschicht liegt in Übereinstimmung mit der Angabe von Autrum in der Größenordnung von $100 \mathrm{mV}$.

Obigen Werten entspricht in der Abb. 3 die Kurve mit dem Parameter $K=0,27$, die ihr Maximum bei $\lambda=5050 \AA$ hat.

Multipliziert man nun die Quanten-Ausbeute $\delta$ für die einzelnen Wellenlängen mit den entsprechenden Werten $\bar{n}$ der notwendig absorbierten Quanten, so ergeben sich folgende Zahlen für die freigemachten Elektronen. 


\begin{tabular}{|c|c|c|c|c|c|c|}
\hline$\lambda[\AA]$ & 4415 & 5050 & 5280 & 5550 & 5880 & 6290 \\
\hline$\delta \bar{n}$ & 5,1 & 3,1 & 4,0 & 4,7 & 4,4 & 4,1 \\
\hline
\end{tabular}

Diese Werte zeigen nur geringe Abweichungen voneinander bis auf den Wert bei $\lambda=5050 \AA$. Ohne den letzteren ergibt sich daraus ein Mittelwert von 4,5 Elektronen. Das bedeutet:

Um eine Sehwahrnehmung auszulösen, müssen an der Sehschwelle die in den Sehzellen absorbierten Quanten eine konstante, von der Wellenlänge des einfallenden Lichtes unabhängige Anzahl von Elektronen freimachen. Es wird also ein von der Wellenlänge unabhängiger Photostrom erzeugt. Diese
Modellvorstellung dürfte auf Grund obiger Überlegungen ihre Berechtigung haben.

Die gleichen Überlegungen und Rechnungen wurden auch für die fovealen Sehzellen durchgeführt. Dabei ergibt sich kein konstanter Wert für das Produkt $\delta \cdot \bar{n}$; vielmehr schwankt er von 6,2 bis 2,2 . Man könnte nun in Verbindung mit der Dreifarben-Theorie an drei Photozellen mit spektral verschiedener Quantenausbeute denken, deren Summenwirkung sich in der gemessenen Empfindlichkeitskurve der fovealen Sehzellen ausdrückt.

Die Ergebnisse lassen es nicht zu, näher auf diese Vorstellung einzugehen. Ich glaube aber, daß eine Erklärung für das Zustandekommen des fovealen Sehvorganges ohne gleichzeitige Deutung des Farbensehens unmöglich ist.

\title{
Versuche zur Synthese des „Steranthrens“, II
}

\author{
Von Heinz Dannenberg \\ Aus dem Max-Planck-Institut für Biochemie, Tübingen \\ (Z. Naturforschg. 9 b, 16-21 [1954]; eingegangen am 15. September 1953)
}

Für den hypothetischen Kohlenwasserstoff „Steranthren“, der in einer 1.2-BenzanthracenForm (II a) und in einer 3.4-Benzphenanthren-Form (II b) existieren müßte, und für die um 2 H-Atome ärmeren "Dehydro-steranthrene" (XIII a-e) werden an Hand des vorliegenden Vergleichsmaterials der Literatur die Isomeriemöglichkeiten und die krebserzeugende Wirkung diskutiert. Während es sich bei den beiden Formen des Steranthrens um eine echte Isomerie handeln muß, ist eine solche bei den Dehydro-steranthrenen unwahrscheinlich. Die entwickelten Vorstellungen sollen durch experimentelle Arbeiten geprüft werden.

$\mathrm{I}^{\mathrm{n}}$

der 1 . Mitteilung ${ }^{1}$ dieser Reihe wurde der Vorschlag gemacht, dem Grundkohlenwasserstoff II den Namen "Steranthren“ zu geben und die von B ergmann ${ }^{2}$ postulierte Verbindung I, deren Bildung aus Cholesterin denkbar ist, als 3.6-Dimethylsteranthren zu bezeichnen.

Steranthren der Zusammensetzung $\mathrm{C}_{23} \mathrm{H}_{18}$ enthält als charakteristische Gruppierung seines Ringsystems die Perinaphthan-Anordnung ${ }^{3}$ der Ringe B, C und D bzw. die Aceperinaphthan-Anordnung ${ }^{4}$ der Ringe A, $\mathrm{B}, \mathrm{C}$ und D (II a), es sind daher zwei isomere Kohlenwasserstoffe (II a und b) denkbar, die sich durch

1 H. D a nnenberg, Liebigs Ann. Chem. 568, 100 [1950].

2 W. B erg m a n n, Z. Krebsforsch. 48, 546 [1939].

3 Zur Nomenklatur des Perinaphthans s. F. F i e s e r u. E. B. H e r s h berg, J. Amer. chem. Soc. 60, 1659 [1938].

4 Zur Nomenklatur des Aceperinaphthans s. H. D a n ne n berg u. D. Da n e n berg-von Dresler, III. Mitt., Liebigs Ann. Chem. (im Druck). die Lage der aromatischen Ringe im Molekül voneinander unterscheiden. Der Kohlenwasserstoff II a
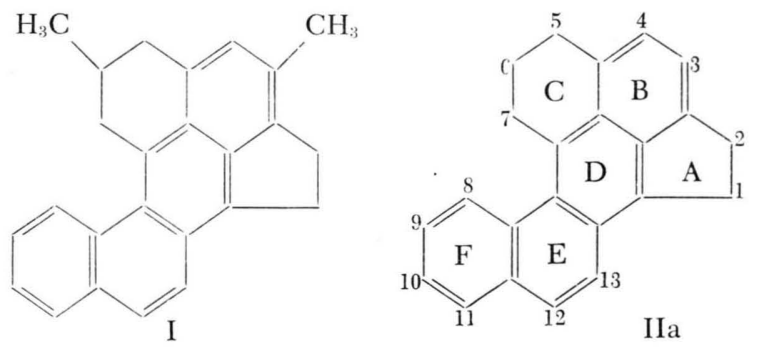<smiles></smiles> 